\title{
A sheep model of cystic fibrosis generated by CRISPR/Cas9 disruption of the CFTR gene
}

\author{
Zhiqiang Fan, ${ }^{1}$ Iuri Viotti Perisse, ${ }^{1}$ Calvin U. Cotton, ${ }^{2}$ Misha Regouski,, Qinggang Meng, ${ }^{1}$ \\ Chaim Domb, ${ }^{2}$ Arnaud J. Van Wettere, ${ }^{1}$ Zhongde Wang, ${ }^{1}$ Ann Harris, ${ }^{3}$ Kenneth L. White, ${ }^{1}$ \\ and Irina A. Polejaeva' \\ 'Department of Animal, Dairy and Veterinary Sciences, Utah State University, Logan, Utah, USA. ${ }^{2}$ Departments of \\ Pediatrics, Physiology and Biophysics, and ${ }^{3}$ Department of Genetics and Genome Sciences, Case Western Reserve \\ University School of Medicine, Cleveland, Ohio, USA.
}

Cystic fibrosis (CF) is a genetic disease caused by mutations in the CF transmembrane conductance regulator (CFTR) gene. The major cause of limited life span in CF patients is progressive lung disease. CF models have been generated in $\mathbf{4}$ species (mice, rats, ferrets, and pigs) to enhance our understanding of the CF pathogenesis. Sheep may be a particularly relevant animal to model CF in humans due to the similarities in lung anatomy and development in the two species. Here, we describe the generation of a sheep model for CF using CRISPR/Cas9 genome editing and somatic cell nuclear transfer (SCNT) techniques. We generated cells with CFTR gene disruption and used them for production of CFTR $^{-1-}$ and CFTR $^{+/-}$lambs. The newborn CFTR $^{-1-}$ sheep developed severe disease consistent with CF pathology in humans. Of particular relevance were pancreatic fibrosis, intestinal obstruction, and absence of the vas deferens. Also, substantial liver and gallbladder disease may reflect CF liver disease that is evident in humans. The phenotype of CFTR $^{-1-}$ sheep suggests this large animal model will be a useful resource to advance the development of new CF therapeutics. Moreover, the generation of specific human CF disease-associated mutations in sheep may advance personalized medicine for this common genetic disorder.

Authorship note: Zhiqiang Fan and luri Viotti Perisse are co-first authors.

Conflict of interest: The authors have declared that no conflict of interest exists.

Submitted: July 13, 2018 Accepted: August 17, 2018 Published: October 4, 2018

Reference information: JCI Insight. 2018;3(19):e123529. https://doi.org/10.1172/jci. insight.123529.

\section{Introduction}

The common inherited disorder cystic fibrosis (CF) is life-shortening, and despite recent major advances in pharmacological treatments for some patients, it remains a focus of intensive investigation (reviewed in ref. 1). Key aspects of human CF pathology include early destruction of the pancreas, likely commencing when pancreatic acini become functional in utero; intestinal dysfunction and potential obstruction; and recurrent lung infection and inflammation, with associated tissue damage and fibrosis (reviewed in ref. 1). Pulmonary disease is the major cause of reduced life span and thus the main focus of current therapeutic approaches. One impediment to advancing the understanding of disease mechanisms and developing new treatments is the fact that no single CF animal model exactly recapitulates all aspects of the disease phenotype in humans (2), though each one has provided new insights. Of particular importance is the etiology of early lung pathology, which is likely triggered by functional inactivation of the CF transmembrane conductance regulator protein (CFTR) early in life. Since aspects of human lung development are not accessible for study in vivo (3), availability of a CF sheep could be a valuable asset to reveal key events in the human CF lung. The focus on sheep as a large animal model of CF was based on the fact that current models of human lung development rely heavily on seminal work in the sheep lung carried out many decades ago (reviewed in ref. 4) (5). Moreover, the developmental time course of CFTR gene expression in the sheep lung appears similar to limited observations in humans (6-9). Further, in addition to the CF pig, another large animal model of CF would be useful for testing new pharmacological therapies for the disease. Earlier attempts to use somatic cell cloning to generate an ovine model of CF were not pursued due to the observed inefficiency of homologous recombination in sheep fibroblasts (10). Here we use highly efficient CRISPR/Cas9 technology to introduce mutations in the CFTR locus of ovine fetal fibroblasts, followed by somatic cell nuclear transfer (SCNT) that led 
A

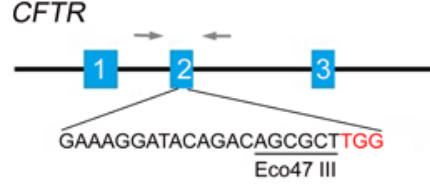

C

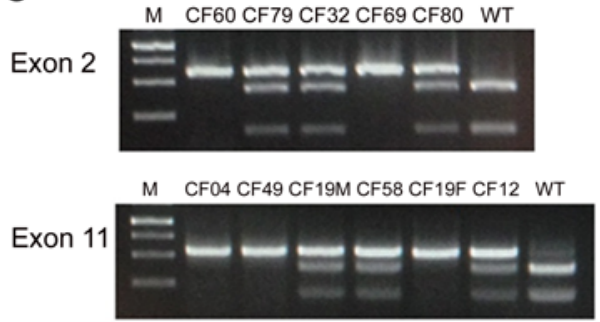

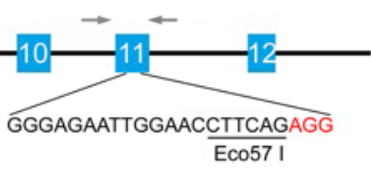

B bp $\quad$ M Tar1 Con1 Tar2 Con2

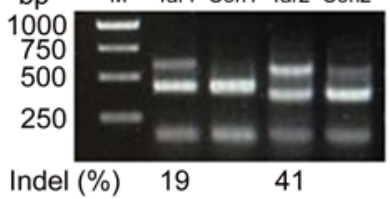

WT CF60 $(\mathrm{M},--)$ ATTTTGAAGAAAGGATACAGACAGCCGCTTGGAATTGTCAG $(+1 \mathrm{nt})$ CF79 $(\mathrm{M},+/-)$ ATTTTGAAGAAAGGATACAGACAGCAGCTTGGAATTGTCAG $(+1 \mathrm{nt})$ CF32 $(\mathrm{M},+/-)$ ATTTTGAAGAAAGGATACAGACAGCCGCTTGGAATTGTCAG $(+1 \mathrm{nt})$ CF69 $(\mathrm{F},-/)$ ATTTTGAAGAAAGGATACAGACAGCCGCTTGGAATTGTCAG $(+1 \mathrm{nt})$ CF80 $(\mathrm{F},+/-)$ ATTTTGAAGAAAGGATACAGACAGCAGCTTGGAATTGTCAG $(+1 \mathrm{nt})$ WT GATGATTATGGGAGAATTGGAACCTTCAGAGGGTAAAATTA CF04 (M, - - -) GATGATTATGGGAGAATTGGAACCT-CAGAGGGTAAAATTA (-1nt) CF49 $(\mathrm{M},-/-)$ GATGATTATGGGAGAATTGGA $\ldots$....... GAGGGTAAAATTA (-7nt) CF19M $(\mathrm{M},+/-)$ GATGATTATGGGAGAATTGGAACCT-CAGAGGGTAAAATTA (-1nt) CF58 (M, +/-) GATGATTATGGGAGAATTGGAACCTCCCAGAGGGTAAAATTA $(+1 \mathrm{nt}, \mathrm{T}-\mathrm{CC})$ CF19F $(F,-\infty)$ GATGATTATGGGAGAATTGGAACC- -CAGAGGGTAAAATTA (-2nt) CF12 (F, +/-) GATGATTATGGGAGAATTGGAACCT-CAGAGGGTAAATTA $(-1 \mathrm{nt})$

Figure 1. Generation of CFTR ${ }^{-/-}$and CFTR $^{+/-}$sheep fetal fibroblast colonies for somatic cell nuclear transfer by CRISPR/Cas9. (A) Schematic diagram of the CFTR targeting sites. The single-guide RNA target sequences for each locus are depicted, with the restriction enzyme recognition sites used for the PCR/restriction fragment length polymorphism (RFLP) assays underlined. Letters in red indicate the protospacer-adjacent motifs (PAMs). Arrows indicate locations of PCR primers. (B) Gene targeting efficiency analysis of 2 targeting vectors at CFTR loci in sheep fetal fibroblasts (SFFs) detected by PCR/RFLP assays. M, 1-kb DNA ladder; Con, control (WT SFFs); Tar, SFFs transfected with each targeting vector. The targeted alleles lost restriction sites through error-prone non-homologous end joining (NHEJ) following Cas9-mediated double-stranded DNA breaks. The mutation efficiency (indels) for target 1 was $19 \%$ and for target 2, 41\%. (C) PCR/RFLP assays for detection of CFTR ${ }^{-/-}$and CFTR ${ }^{+/-}$single-cell-derived SFF colonies with mutations at exon 2 (upper panel) or exon 11 (lower panel) of the CFTR gene. (D) Sequencing analysis of CFTR ${ }^{-/-}$and CFTR ${ }^{+/-}$colonies. Letters in yellow indicate the nucleotides inserted at cleavage sites. M, male; F, female; -/-, both alleles targeted: +/-, single allele targeted; -1nt, 1-nucleotide deletion; +1nt, 1-nucleotide insertion.

to the production of $C F T R^{-/-}$and $C F T R^{+/-}$lambs. These lambs present a severe phenotype with multiple organ involvement, including intestinal obstruction, pancreatic fibrosis, absence of vas deferens, and liver disease, which is consistent with CF pathology in humans.

\section{Results}

Generation of CFTR $^{-/-}$and CFTR ${ }^{+/-}$fetal fibroblasts using CRISPR/Cas9. We used primary fetal fibroblasts from domestic sheep (Ovis aries), since they are the cells of choice for transgenic SCNT (11). Specific PCR primers were designed according to the sheep CFTR genome sequence (GenBank, NC_019461.2) and used to amplify exon 2 (F: AGATGCCTTACTTTATTCAC, R: CCAACTGCCTTTACCCTA, 625bp) and exon 11 (F: GCATAGCAGCATACCCAA, R: GTAACCAAACCAGCCCAC, 612bp) and partial intron sequences. The choice of these exons was determined by future plans to generate human-specific disease-associated mutations in these exons. We designed CRISPR/Cas9 targeting sites based on exon 2 and 11 sequences, and specific restriction enzyme sites were introduced to target loci in order to facilitate mutation detection (Figure 1A). A pair of oligonucleotides (oligos) for each targeting site was synthesized and ligated to the pX330 vector (Addgene plasmid 42230; Table 1) as previously described (12). Sheep fetal fibroblasts (SFFs) at early passages were transfected with the targeting vectors using an electroporation-based method, and gene mutation efficiency was determined by PCR/restriction fragment length polymorphism (RFLP) assays 3 days after transfection. The mutation efficiency of targeting vectors 1 and 2 was $19 \%$ and $41 \%$, respectively (Figure 1B). Single-cell-derived CFTR-mutated fibroblast colonies were isolated by limiting dilution

Table 1. DNA oligonucleotides for the construction of CRISPR/Cas9 targeting vectors

\begin{tabular}{ccc}
\hline Name & Gene/exon & Sequence (5'-3') \\
Cri-sgRNA-2F & CFTR/exon 2 & CACCGAAAGGATACAGACAGCGCT \\
Cri-sgRNA-2R & CFTR/exon 2 & AAACAGCGCTCTCTCTATCCTTTC \\
Cri-sgRNA-4F & CFTR/exon 11 & CACCGGGAGAATTCGAACCTTCAG \\
Cri-sgRNA-4R & CFTR/exon 11 & AAACCTCAAGGTTCCAATTCTCCC
\end{tabular}


Table 2. Isolation of sheep $\mathrm{CFTR}^{-/-}$and $\mathrm{CFTR}^{+/-}$fibroblast colonies

\begin{tabular}{|cccccc}
\hline Cell line /sex & Targeting vector/exon & No. of colonies isolated & $\begin{array}{c}\text { No. of colonies with } \\
\text { mutations(\%) }\end{array}$ & Biallelic disruption (\%) & Monoallelic disruption (\%) \\
\hline SFF5/M & Tar1/exon 2 & 51 & $10(19.6)$ & $5(9.8)$ & $5(9.8)$ \\
SFF4/M & Tar2/exon 11 & 49 & $17(34.7)$ & $13(26.5)$ & $4(8.2)$ \\
SFF3/F & Tar1/exon 2 & 87 & $6(6.9)$ & $4(4.6)$ & $2(2.3)$ \\
SFF3/F & Tar2/exon 11 & 65 & $5(7.7)$ & $3.6)$ & $2(3.1)$
\end{tabular}

SFF, sheep fetal fibroblast; $M$, male; F, female.

and screened by PCR/RFLP assays (Figure 1C). Targeted CFTR disruption was achieved in both male and female colonies for exons 2 and 11 at various efficiencies ranging between $6.9 \%$ and 34.7\% (Table 2). Sequence analysis of the PCR products indicated that small insertions and deletions (indels) were introduced at each of the targeted CFTR loci in these colonies (Figure 1D).

Generation of $\mathrm{CFTR}^{-/-}$and $\mathrm{CFTR}^{+/-}$sheep by SCNT. Fetal fibroblasts homozygous or heterozygous for CFTR mutations were used as donor cells for SCNT. In total 1,029 cloned embryos were transferred into 73 estrus-synchronized recipients. Male and female lambs were produced for each group (Table 3). PCR/RFLP and sequence results indicated that all cloned fetuses/lambs carried the same mutations as those of the donor cells they originated from (Figure 2, B and $C)$. No difference $(P>0.05)$ was found in pregnancy and term development rates between $\mathrm{CFTR}^{-/-}$and $\mathrm{CFTR}^{+/-}$groups. Late-term abortions and stillborn lambs were observed in both groups (5 of 17 in the $C F T R^{-/-}$group and 3 of 16 in $C F T R^{+/-}$). Additionally, body weights were similar in the $C F T R^{-/-}$and $C F T R^{+/-}$groups at birth $(6.79 \pm 0.61 \mathrm{~kg}$ and $6.12 \pm 0.37 \mathrm{~kg}$, respectively; $P=0.36)$. Live-born $C F T R^{-/-}$lambs were considered not viable due to severe intestinal obstruction (meconium ileus) and so were euthanized within 48 hours after birth. Two of $13 \mathrm{CFTR}^{+/}$lambs were euthanized due to broken ribs (resulting from accidental injury by surrogate mothers), and one more developed septic arthritis due to an umbilical bacterial infection. The rest of the $C F T R^{+/-}$sheep appeared to be healthy and have developed to adulthood (Figure 2A).

Gross and histological characterization of the $C F T R^{-1-}$ and $C F T R^{+/-}$lambs. We performed a detailed histological evaluation on $15 \mathrm{CFTR}^{-/-}$newborn lambs (0-3 days old) and on 5 CFTR ${ }^{+/-}$animals (4 at 0-3 days and 1 at 57 days). The following organs were assessed: trachea, lung, intestinal tract, forestomach, pancreas, liver, thyroid gland, heart, kidney, urinary bladder, thymus, spleen, umbilicus, skeletal muscle, and brain. Additionally, male genital ducts, epididymis, and vas deferens were evaluated in the $C F T R^{-/-}$and $C F T R^{+/-}$males, and ovary and uterus for the $C F T R^{-/-}$and $C F T R^{+/-}$females and compared with those of the WT controls $(n=3)$. The histopathological findings are summarized in Table 4.

Intestine. The most overt phenotype associated with loss of CFTR in the sheep was profound intestinal obstruction, similar to the meconium ileus phenotype seen in some $(\sim 15 \%)$ babies with $C F$, which was evident in $100 \%$ of $\mathrm{CFTR}^{-/-}$newborn lambs (Table 4 and Figure 3). A relatively sharp demarcation was evident

Table 3. Development rates following SCNT using $\mathrm{CFTR}^{-/-}$and $\mathrm{CFTR}^{+/-}$fibroblasts

\begin{tabular}{|c|c|c|c|c|c|c|c|}
\hline $\begin{array}{l}\text { KO colonies } \\
\text { (sex, genotype) }\end{array}$ & Cell line & Exon targeted & $\begin{array}{l}\text { No. of embryos } \\
\text { transferred }\end{array}$ & Pregnancy rate (\%) & Term rate (\%) & $\begin{array}{l}\text { No. lambs born } \\
\text { alive/total* (\%) }\end{array}$ & $\begin{array}{l}\text { No. lambs alive at } \\
1 \text { month (\%) }\end{array}$ \\
\hline CF60 (M, -/-) & SFF5 & Exon 2 & 262 & $13 / 20(65.0)$ & $11 / 20(55.0)$ & $11 / 15$ (73.3) & $0 / 11$ \\
\hline CF49 (M, -/-) & SFF4 & Exon 11 & 99 & $0 / 7(0)$ & $0 / 7(0)$ & NA & NA \\
\hline CF19F (F, -/-) & SFF3 & Exon 11 & 134 & $5 / 9(55.6)$ & $1 / 9(11.1)$ & $1 / 2(50.0)$ & $0 / 1$ \\
\hline CFTR $^{-/-}$total & & & 495 & $18 / 36(50.0)^{A}$ & $12 / 36(33.3)^{\mathrm{A}}$ & $12 / 17(70.6)^{A}$ & $0 / 12(0)^{A}$ \\
\hline CF79 (M, +/-) & SFF5 & Exon 2 & 117 & $2 / 9(22.2)$ & $1 / 9(11.1)$ & $0 / 1(0)$ & NA \\
\hline CF19M $(M,+/-)$ & SFF4 & Exon 11 & 166 & $5 / 10(50.0)$ & 4/10 (40.0) & $5 / 6(83.3)$ & 4/5 (80.0) \\
\hline CFTR $^{+/-}$total & & & 534 & $16 / 37(43.2)^{A}$ & $14 / 37(37.8)^{A}$ & $13 / 16(81.3)^{A}$ & $11 / 13(84.6)^{\mathrm{B}, * *}$ \\
\hline
\end{tabular}

${ }^{*}$ Eight twin pregnancies were obtained (4 in CFTR ${ }^{-/-}$and 4 in CFTR ${ }^{+/}$groups). ${ }^{* *}$ Two CFTR ${ }^{+/-}$lambs were euthanized due to broken ribs resulting from accidental injuries. Values in the same column with different superscript letters differ significantly $\left(P<0.05, \chi^{2}\right.$ test). 
A

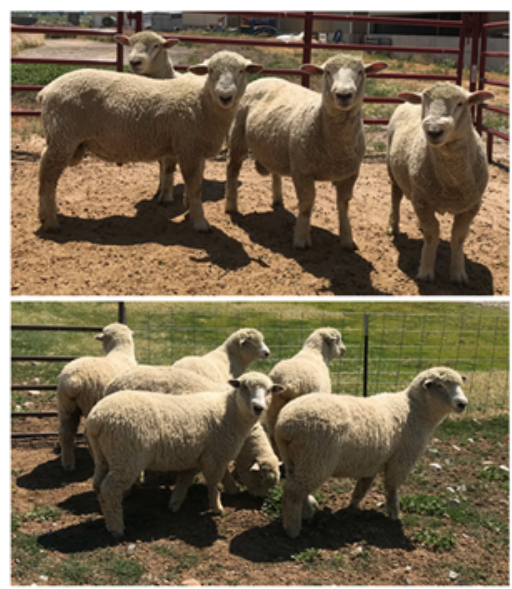

B

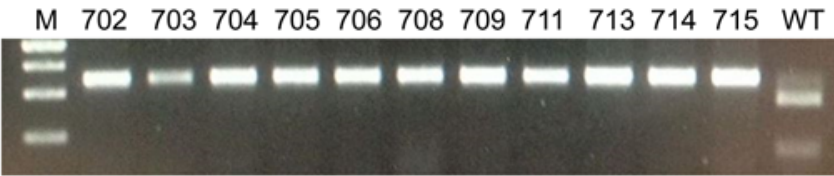

M 716723725726727728729730733 WT

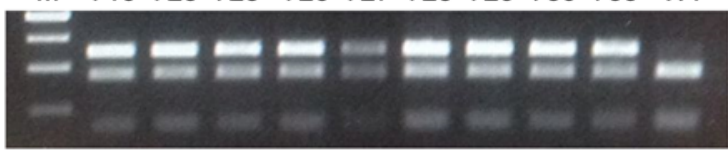

M 724717718719720721 WT

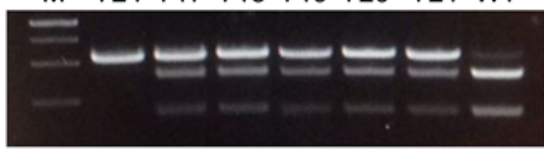

C

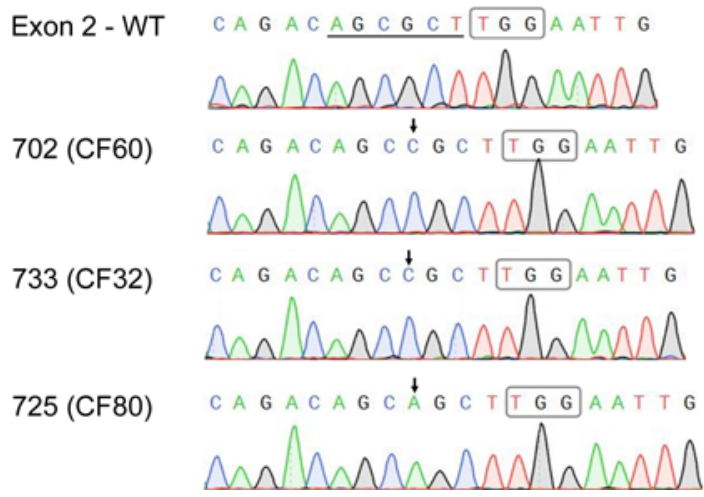

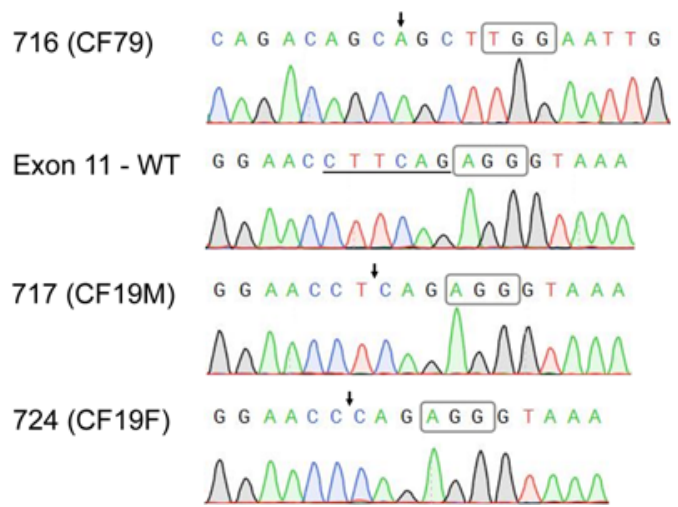

Figure 2. Detection of CFTR mutations in the cloned lambs by PCR/restriction fragment length polymorphism assays and Sanger sequencing. (A) Cloned CFTR ${ }^{+/-}$sheep at 13-16 months of age: 4 males (top) and 6 females (bottom). (B) Identification of CFTR ${ }^{-/-}$and CFTR ${ }^{+/-}$cloned lambs with mutations in exon 2 (upper and middle panels) or exon 11 (lower panel) by PCR/restriction fragment length polymorphism (RFLP) assays (702-706, 708, 709, 711, 713715, and 724 are CFTR ${ }^{-1-} ; 716-721,723,725-730$, and 733 are CFTR ${ }^{+/}$. (C) Representative sequence analysis for CF lambs. In total, 6 CFTR-targeted colonies (4 colonies with mutations in exon 2 and 2 in exon 11) were successfully used for production of CFTR ${ }^{-/-}$or CFTR ${ }^{+/-}$cloned sheep (Table 3). Representative images show sequence results for one animal obtained from each of the donor cell colonies used in the study. Exon 2 and 11 WT sequences are also shown. Restriction enzyme recognition sites used for the PCR/RFLP assays are underlined. Arrows indicate the mutation sites. The protospacer-adjacent motif (PAM) sequences used for Cas9 targeting are indicated by boxes.

between a distended oral intestine and small-diameter aboral small intestine or colon (Figure 3B). Obstruction was predominately seen in the distal jejunum (12 of 15$)$ or in the proximal, mid-, or distal spiral colon (3 of 15). The intestine oral to the obstruction site was distended with a large amount of meconium (Figure $3 \mathrm{D}$ ), and the intestinal diameter ranged from 1 to $2.5 \mathrm{~cm}$. The intestine and/or colon aboral to the obstruction site had a small diameter $(3-5 \mathrm{~mm})$ and contained thick mucoid content. Distended colonic glands associated with buildup of mucoid material in the lumen were seen in $\mathrm{CFTR}^{-/-}$colon sections (Figure 3, F and $\mathrm{H}$ ) compared with normal control (Figure 3, E and G). No intestinal lesions were observed in the heterozygous and control lambs (normal small intestinal diameter was $0.6-1 \mathrm{~cm}$ and colon diameter 1-1.5 cm).

Pancreas. The pancreatic pathology of the $C F T R^{-/-}$sheep was complex, as it may have reflected both a cloning artifact (caused by incomplete reprogramming during SCNT) and a phenotype resulting from loss of CFTR (Figure 4). First, variable pancreatic hypoplasia or aplasia was evident in both homozygous (11 of 15) and heterozygous (3 of 5) lambs and therefore may be SCNT-related. Pancreas was not grossly visible in 7 of 15 homozygous and 3 of 5 heterozygous lambs; and was approximately one-fifth to two-thirds of expected size in 4 of 15 homozygous lambs. Pancreatic tissue or tissues where the pancreas should be located were collected for histology. No pancreatic tissue was detected on histology in 6 lambs that had no grossly visible pancreas (3 of $7 \mathrm{CFTR}^{-/-}$and 3 of $4 \mathrm{CFTR}^{+-}$). However, in addition to the hypoplasia, pancreatic fibrosis was evident in $40 \%$ of $\mathrm{CFTR}^{-/}$animals and none of the heterozygotes, and so was considered a phenotype resulting from loss of CFTR function (Figure 4, B, C, E, and F). Among $7 \mathrm{CFTR}^{-/}$lambs without a visible pancreas, based 
Table 4. Histopathological evaluation of $\mathrm{CFTR}^{-/-}$and $\mathrm{CFTR}^{+/-}$lambs

\begin{tabular}{|c|c|c|c|c|c|c|c|}
\hline & Small intestine & Pancreas & Liver & Gallbladder & Vas deferens & Lung & Kidney \\
\hline CFTR $^{-/-}(n=15)$ & $\begin{array}{l}\text { Obstruction, } \\
\text { meconium ileus } \\
15 / 15(100 \%)\end{array}$ & $\begin{array}{c}\text { Hypoplasia or } \\
\text { aplasia } 11 / 15 \\
(73.3 \%) \text { Fibrosis } \\
6 / 15(40 \%)\end{array}$ & $\begin{array}{l}\text { Periportal fibrosis } \\
11 / 14(78.6 \%) \\
\text { intrahepatic } \\
\text { cholestasis } 12 / 14 \\
(85.7 \%)^{A}\end{array}$ & $\begin{array}{l}\text { Hypoplasia } \\
12 / 15 \text { (80\%) }\end{array}$ & $\begin{array}{l}\text { Aplasia or atrophy } \\
12 / 12(100 \%)\end{array}$ & No lesions & $\begin{array}{c}\text { Hydronephrosis } \\
13 / 15(86.7 \%)\end{array}$ \\
\hline $\mathrm{CFTR}^{+/-}(n=5)^{\mathrm{B}}$ & No lesions & $\begin{array}{c}\text { Hypoplasia or } \\
\text { aplasia, } 3 / 5\end{array}$ & No lesions & Normal size & Normal & No lesions & $\begin{array}{c}\text { Hydronephrosis } \\
4 / 5(80 \%)\end{array}$ \\
\hline
\end{tabular}

$(60 \%)$

${ }^{A}$ Liver of 1 of the $C F T R^{-/-}$lambs was not evaluated due to severe autolysis. ${ }^{B}$ Four CFTR ${ }^{+/-}$lambs subjected to histopathology were 0-3 days old, and the 5th lamb was 57 days old. Ten additional CFTR $^{+/}$sheep produced in the study were 13-15 months old at the time of preparation of the manuscript and appeared to be healthy.

on gross morphology, some residual pancreatic tissue was evident on histology. Three showed mild multifocal to severe diffuse fibrosis with acinar loss and ductal dilation. A fourth was not examined histologically due to autolysis. Two homozygous CF-null lambs with pancreases two-thirds of the expected size also had moderate, multifocal interstitial fibrosis, and acinar and ductal dilation. Additionally, 1 of the $4 C^{-1} F^{-1}$ lambs with grossly normal pancreases also had mild multifocal interstitial fibrosis. No fibrotic lesions were observed in the heterozygote or control lambs.

Liver and gallbladder. In homozygous $C F T R^{-/-}$lambs, the liver was of normal size, shape, and consistency, with a color varying from mahogany, the normal color, to yellow-brown. In contrast, the gallbladder in 12 of 15 animals was one-half to one-third the expected size. Gross examination revealed that the gallbladder was either empty (9 of 15), contained a small amount of bile ( 3 of 15), or contained a normal amount of unusually viscous bile (1 of 15). In $1 \mathrm{CFTR}^{-/-}$animal, an expected amount of normal bile was observed. On histology, severe intrahepatic cholestasis (12 of 15 animals), marked hepatocellular glycogen accumulation ( 3 of 15$)$, and/or portal fibrosis and biliary hyperplasia (10 of 15$)$ resembling biliary fibrosis were present in $\mathrm{CFTR}^{-/-}$newborn lambs (Figure 5, B, C, E, and F). Livers from $\mathrm{CFTR}^{-/-}$newborn lambs were characterized by bile duct proliferation and fibrosis in the portal areas, consistent with biliary fibrosis/cirrhosis (Supplemental Figure 1; supplemental material available online with this article; https://doi. org/10.1172/jci.insight.123529DS1). Hepatocellular vacuolation was determined to be due to glycogen accumulation, as the hepatocellular vacuoles stained with periodic acid-Schiff (PAS) - but not after diastase treatment - or with oil red O (lipid stain). No liver lesions were observed in the heterozygous and control lambs (data not shown).

Male genital ducts: epididymis and vas deferens. None of the homozygous $C_{F T R^{-1}}$ males $(n=12)$ had a grossly visible or histologically detectable vas deferens, though the majority of the epididymis was grossly and histologically evident. All 3 heterozygous male lambs and 3 control male lambs had normal genital ducts.

Lung. As summarized in Table 4, the lungs of $C F T R^{-/}$lambs appeared to be grossly normal at birth (Supplemental Figure 2). Though histological examination (not shown) revealed minimal mucosal gland dilation in $60 \%$ of the CFTR-null lambs, this phenotype was also observed in $25 \%$ of heterozygous animals and in 1 of $3 \mathrm{WT}$ control lambs, and so is unlikely to be directly correlated to the loss of CFTR.

Kidney. No specific kidney phenotype was associated with loss of CFTR in the lambs. However, hydronephrosis, a common pathology in sheep $(13,14)$ and cattle $(15,16)$ derived from SCNT was evident in $86.7 \%$ of CFTR $^{-1-}$ lambs and $80 \%$ of CFTR $^{+/-}$animals examined.

Additionally, no lesions were observed in the other tissues evaluated, including heart, thyroid gland, trachea, thymus, spleen, umbilicus, skeletal muscle, forestomach, urinary bladder, testis, ovary, uterus, and brain obtained from either $C F T R^{-/-}$or $C F T R^{+/-}$lambs.

Loss of CFTR channel function was observed in sheep tracheal epithelial cells derived from CFTR-null lambs. We isolated epithelial cells from lamb tracheal segments and established a monolayer culture as described previously for human tracheal epithelial cells (17). Filters were mounted in a temperature-controlled Ussing chamber that allows for the separate perfusion of the apical and basolateral compartments with a Krebs-Ringer bicarbonate solution, as previously described $(17,18)$. Transepithelial voltage was measured and clamped to 0 to measure short-circuit current. The epithelium was periodically voltage 

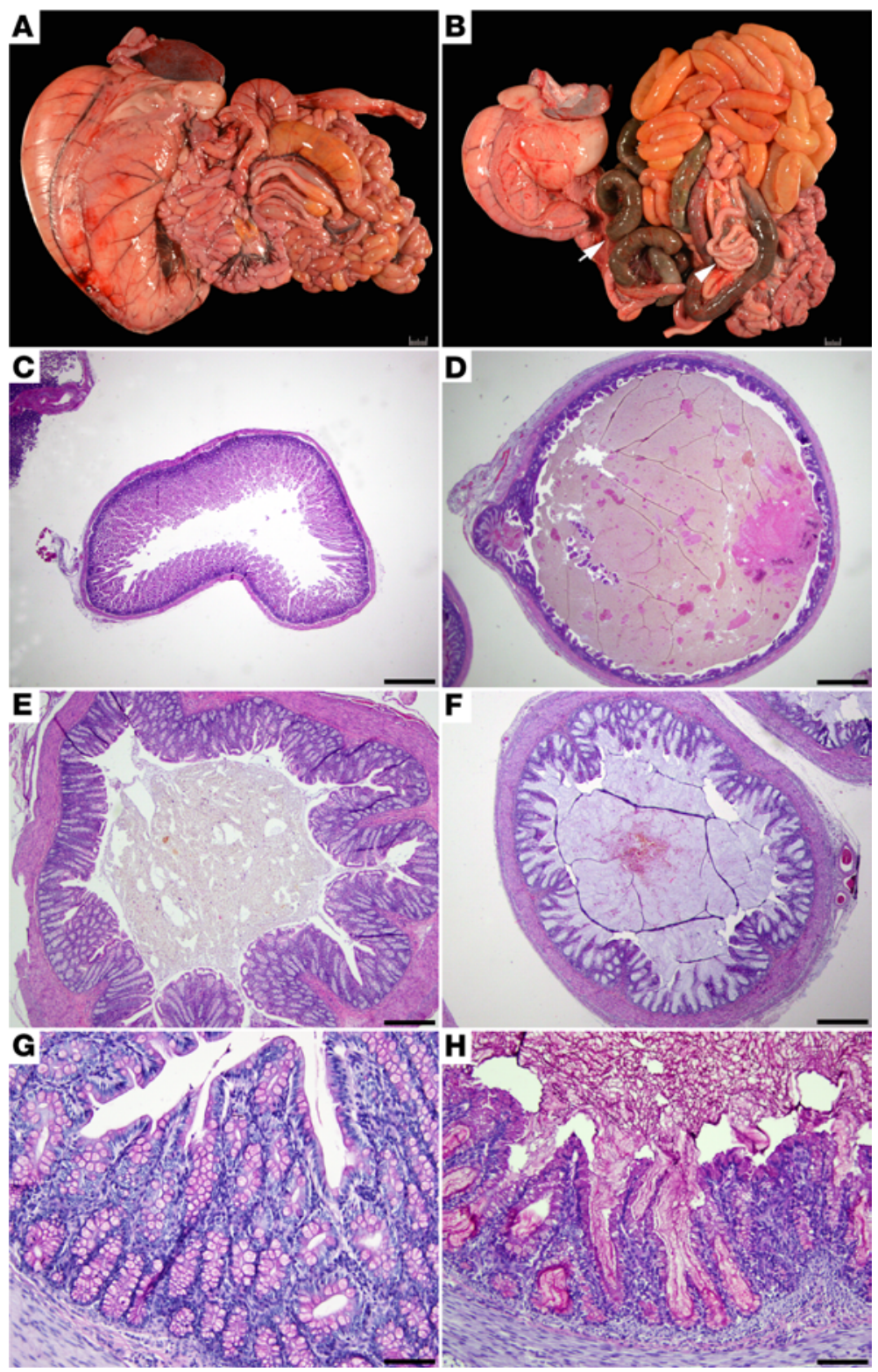

Figure 3. Intestinal pathology of newborn CFTR $^{-/-}$lamb. (A) One-day-old control lamb intestinal tract. (B) Meconium ileus in intestinal tract of a 1-day-old CFTR ${ }^{-/-}$lamb. Small intestine is dilated with meconium (arrow). Colon is plugged with mucus (arrowhead). (C) Control lamb small intestine histology. (D) CFTR ${ }^{-/-}$lamb small intestine is dilated and filled with meconium. (E) Control lamb colon histology. (F) $\mathrm{CFTR}^{-1-}$ colon is filled with large amount of mucus. (C) Control lamb colon histology. (H) CFTR $^{-1-}$ colon with colonic glands filled and distended with large amount of mucus. C-F: H\&E staining. $\mathbf{G}$ and $\mathbf{H}$ : Periodic acid-Schiff (PAS) staining. $\mathbf{C}$ and $\mathbf{D}: \times 20$; scale bars: $1 \mathrm{~mm}$. E and F: ×40; scale bars: $500 \mu \mathrm{m}$. G and $\mathbf{H}$ : $\times 400$; scale bars: $50 \mu \mathrm{m}$.

clamped to non- 0 values to measure transepithelial resistance. After baseline recording, amiloride (100 $\mu \mathrm{M})$ was added to the apical side, followed by addition of forskolin $(10 \mu \mathrm{M})$ to the basal side, and then CFTR inhibitor I172 or GlyH-101 (10 or $20 \mu \mathrm{M}$, respectively) was added to the apical solution (Figure 6). The data shown in Figure 7 are representative of results obtained with 6-8 filters from each of 3 WT lambs and $3 \mathrm{CFTR}^{-/-}$lambs. The baseline short-circuit currents were similar in $\mathrm{CFTR}^{-/-}$and WT cells; however, inhibition of epithelial sodium channel-dependent (ENaC-dependent) sodium absorption with amiloride revealed a distinct difference between mutant and normal cultures. The short-circuit current in $C F T R^{-/-}$cultures fell to values near 0, suggesting that baseline current was entirely dependent on sodium absorption. Subsequent exposure to forskolin (to increase cAMP and activate CFTR) failed to increase current, consistent with a CFTR-knockout phenotype. Exposure to CFTR inhibitor (GlyH-101), as expected, did not alter current. In contrast, when WT cultures were exposed to amiloride, the shortcircuit current was inhibited by $\sim 40 \%$. Subsequent exposure to forskolin caused a small increase in current, and GlyH-101 partially inhibited the short-circuit current. Transepithelial resistance values and the changes in resistance upon exposure to amiloride, forskolin, and GlyH are consistent with short-circuit current responses (data shown in Supplemental Figure 3). The results suggest that CFTR anion channels are constitutively active in WT sheep tracheal cell cultures and are only modestly increased by exposure 

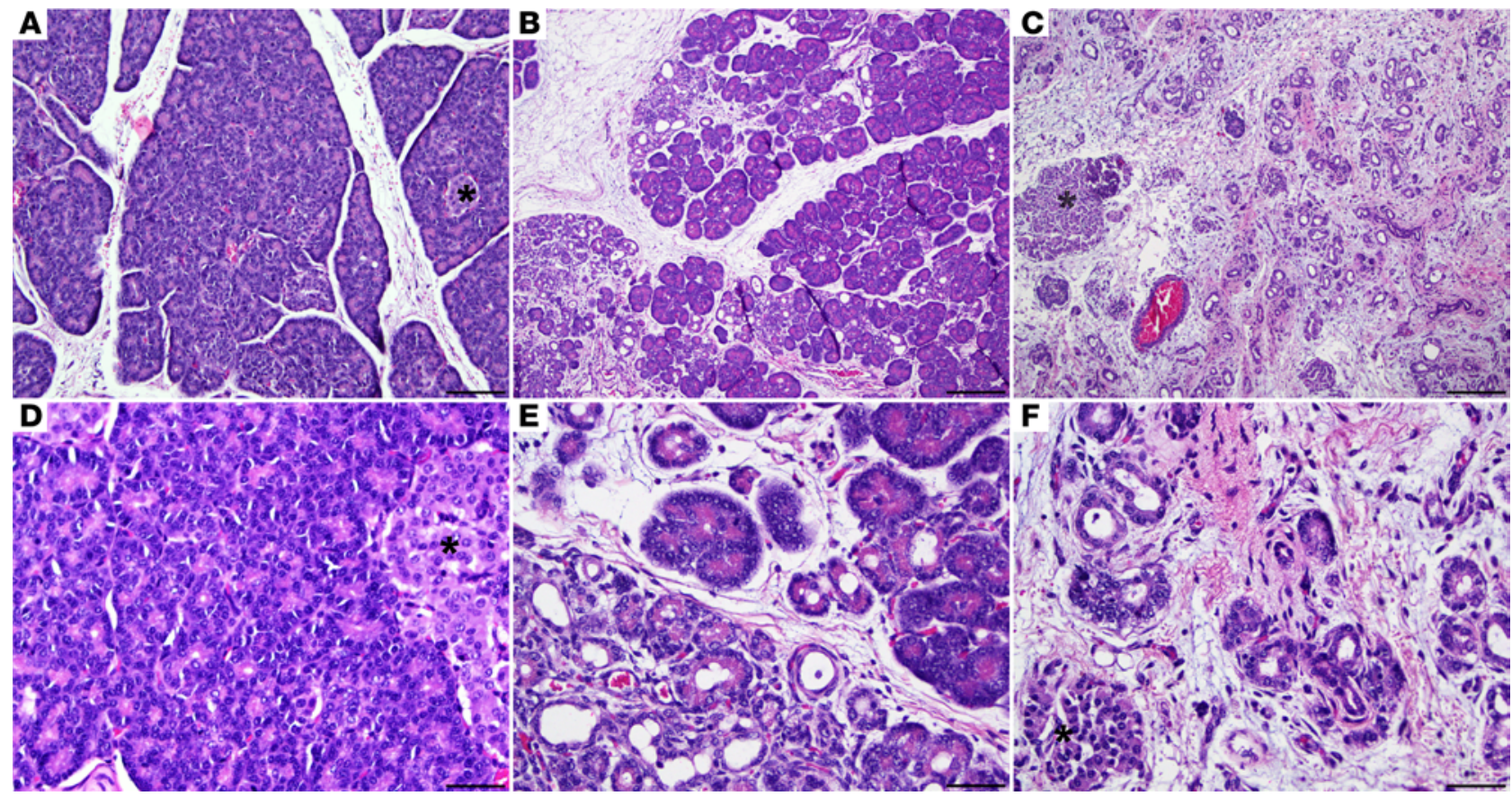

Figure 4. Pancreatic pathology of newborn CFTR ${ }^{-/-}$lambs. (A) Histology of the pancreas of a control lamb. Note the closely packed exocrine pancreatic acini. The asterisk indicates islet of Langerhans. (B) Histology of a CFTR ${ }^{-1-}$ lamb pancreas with mild pancreatic hypoplasia or atrophy and interstitial fibrosis. Note the decreased density and increased space between the exocrine pancreatic acini. (C) Histology of a CFTR ${ }^{-/-}$lamb pancreas with severe pancreatic hypoplasia or atrophy and interstitial fibrosis. Only pancreatic ducts separated by fibrous and adipose tissues are present. (D) Higher magnification of the pancreas of the control lamb in A. (E) Higher magnification of the CFTR ${ }^{-1-}$ lamb pancreas in B. (F) Higher magnification of the CFTR ${ }^{-/-}$lamb pancreas in C. H\&E staining. A-C: $\times 100$, scale bars: $100 \mu \mathrm{m}$. D-F: ×400; scale bars: $50 \mu \mathrm{m}$.

to forskolin. In contrast, CFTR-dependent anion secretion was completely absent from primary tracheal cell cultures derived from $C F T R^{-/}$animals.

Assessment for potential introduction of off-target mutations by CRISPR/Cas9. To examine whether inadvertent off-target (OT) events occurred in the $C F T R^{-/-}$and $C F T R^{+/-}$sheep, we conducted a basic local alignment (BLAST) search of the sheep nucleotide sequence database with CFTR targeting sequences as the queries to find the genomic sequences with the highest homologies. Benchling software was used to predict the possible OT sequences in the sheep genome. Then all potential OT sequences within genes and unknown genome regions that have at least $0.9 \%$ probability to be targeted were selected for testing. In total, we chose 19 potential OT sites (Supplemental Table 1). We subjected these potential OT sites to PCR and Sanger sequencing analysis by using the genomic DNA isolated from $3 \mathrm{CFTR}^{-1}$ and $3 C_{C F T R^{+-}}$sheep: CF713 and CF708 (CFTR ${ }^{-/}$, exon 2), CF724 (CFTR ${ }^{-/}$, exon 11), CF720 and CF717 (CFTR ${ }^{+/}$, exon 11), and CF728 (CFTR ${ }^{+/-}$, exon 2). Our analysis verified that none of the animals had mutations in the analyzed potential OTs (Supplemental Figure 4).

\section{Discussion}

The generation of large farm animals by cloning more than 20 years ago $(19,20)$, opened a new chapter in developing animal models of human genetic disease to advance new therapeutics and provide opportunities to better study disease etiology. $\mathrm{CF}$ is the most common genetic disease among European populations, and though new therapies are effective at treating some mutations in the CFTR locus, no suitable drugs are available for many of the more than 2,000 disease-associated errors reported in the gene. The suggestion that a sheep model of CF might be useful in this regard was also made more than 2 decades ago (21), but this was not realized until the development of CRISPR/Cas9 technologies $(12,22)$ made it possible to easily target the sheep CFTR locus. Here we describe the generation of a $C F T R^{-/-}$sheep derived from CRISPR/Cas9 disruption of the CFTR locus in SFFs, which were subsequently used for cloning of live animals. The phenotype associated with the loss of function of CFTR in the sheep was severe, and as is the case for all other animal models of $\mathrm{CF}$, was dominated by intestinal obstruction. 

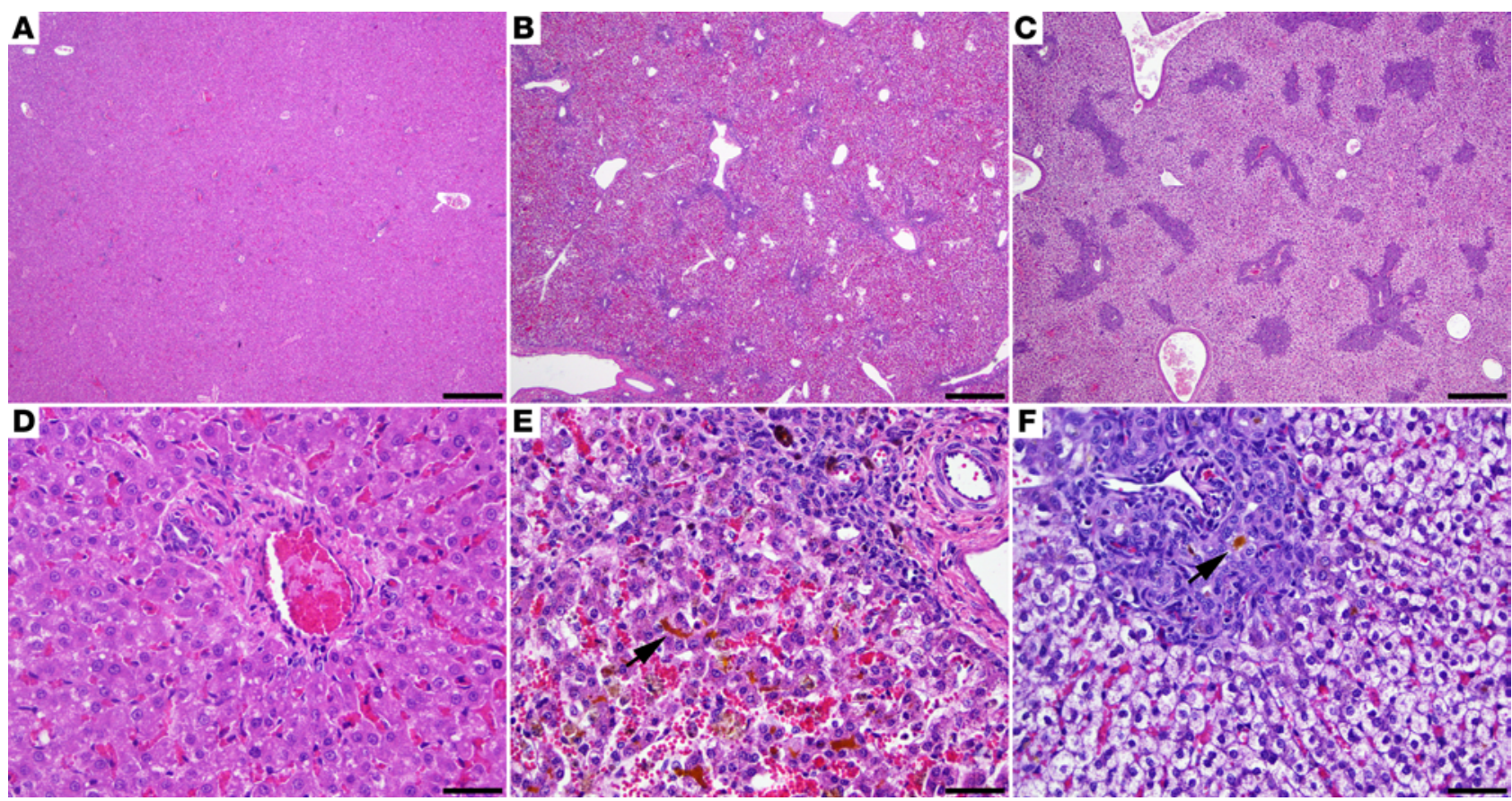

Figure 5. Liver pathology of newborn CFTR ${ }^{-/-}$lambs. (A and D) One-day-old control lamb liver. (B and C) Biliary fibrosis in a 1-day-old CFTR ${ }^{-/-}$lamb liver. Prominent hepatocellular glycogen accumulation was present in some lambs (C). (E and $\mathbf{F}$ ) Close up of the liver with biliary fibrosis. Severe intrahepatic cholestasis is present (arrows). H\&E staining. A-C: $\times 40$; scale bars: $500 \mu \mathrm{m}$. D-F: $\times 400$; scale bars: $50 \mu \mathrm{m}$.

Though this phenotype is managed through dietary changes in $\mathrm{CF}$ rodents, in other models such as the CF pig and CF ferret $(23,24)$ "gut correction" by introduction of a functional CFTR gene driven by an intestine-specific promoter such as the fatty acid binding protein (FABP) has been used to alleviate the intestinal disease $(25,26)$. Like $C F T R^{-/-}$pigs, $100 \%$ of $C F T R^{-/-}$lambs developed intestinal obstruction. Intestinal CFTR expression without pancreatic or hepatic correction was sufficient to rescue meconium ileus in the $\mathrm{CFTR}^{-/-}$pigs (25), and a similar approach is likely to be effective in CF sheep.

A comparison of the phenotype of the CF pig and CF sheep at birth is of particular interest, since due to their size, these 2 models may be the most relevant for performing preclinical trials for promising new drugs or perhaps gene replacement or editing vectors. Of note, many aspects of the CF pig phenotype were revealed by studies over several years after the initial reports of their generation $(23,27)$. This is particularly relevant to lung disease, since they required alleviation or surgical intervention of the intestinal disease to enable survival past the perinatal period. At this point it is too early to know whether the $\mathrm{CF}$ sheep will recapitulate human $\mathrm{CF}$ lung disease over time. Importantly, lung disease in the CF pig is not evident at birth, though animals exhibit an early failure to clear bacteria, and this deficit subsequently causes lung inflammation, tissue remodeling, and mucus accumulation $(23,28)$.

One feature of the CF sheep that is remarkably similar to the human disease is fibrosis of the pancreas, which is already well advanced at birth (29). Further characterization of this defect will depend on natural breeding of the $C F T R^{+/-}$sheep to exclude the impact of pancreatic hypoplasia, which was common in both CFTR-null and -heterozygous animals, and therefore is likely to be associated with SCNT. Newborn CF pigs also show pancreatic inflammation, fibrosis, and abnormal tissue architecture and histology $(30,31)$. They also have liver and gallbladder disease that is more advanced and prevalent than in human CF (32). The liver phenotype of $\mathrm{CFTR}^{-/-}$sheep is also more severe than in humans with $\mathrm{CF}$, where a minority of patients are affected. Nearly $80 \%$ of the CF sheep showed periportal fibrosis with biliary hyperplasia, and more than $85 \%$ had intrahepatic cholestasis, while heterozygote animals showed no liver lesions. Concurrently, $80 \%$ of the CFTR-null sheep had a hypoplastic gallbladder, again a phenotype that was not seen in $C F T R^{+/-}$lambs. This liver and gallbladder phenotype was more frequent and severe in the CFTR-null sheep than the null pigs.

Kidney abnormalities are commonly observed in lambs produced by SCNT and are often characterized by development of hydronephrosis $(13,14)$. Hydronephrosis was evident in $87 \%$ of CFTR $^{-1-}$ lambs and 


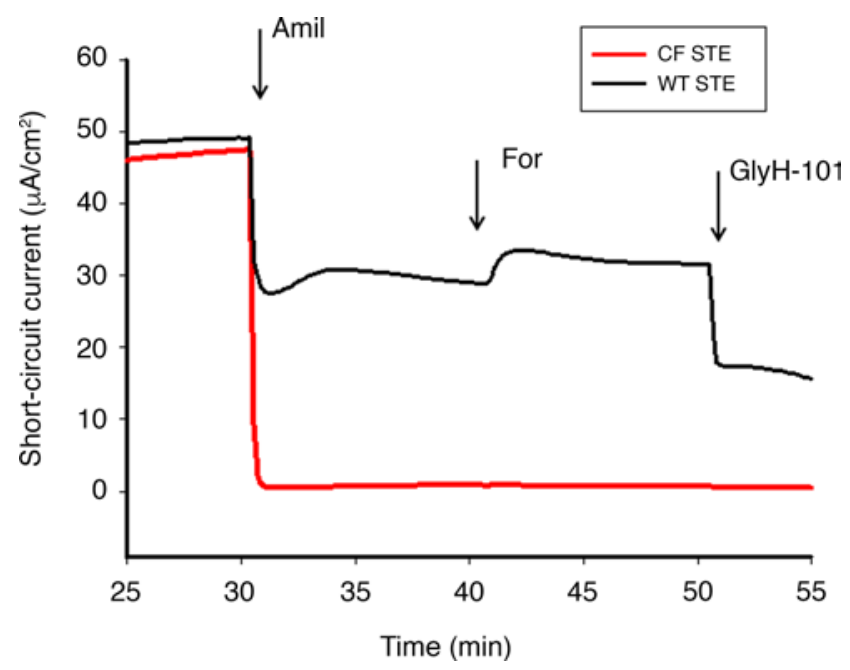

Figure 6. Representative traces of short-circuit current in WT and CFTR ${ }^{-/-}$sheep tracheal epithelial cell cultures. Primary cultures of sheep tracheal epithelial (STE) cells (passage 2) were seeded onto permeable filter supports and maintained at the air-liquid interface for 6 weeks. At the time of measurement, the filter inserts were placed in Ussing chambers and bathed on both sides with Krebs-Ringer bicarbonate solution bubbled with $95 \% \mathrm{O}_{2} / 5 \% \mathrm{CO}_{2}$ and maintained at $37^{\circ} \mathrm{C}$. At the indicated times, amiloride (Amil, $100 \mu \mathrm{M}$, apical), forskolin (For, $10 \mu \mathrm{M}$, basolateral), and GlyH-101 (20 $\mu \mathrm{M}$, apical) were added. CF, CFTR ${ }^{-1-}$.

$80 \%$ of $\mathrm{CFTR}^{+/-}$animals examined, and is not associated with loss of CFTR in the lambs. Natural breeding of $\mathrm{CFTR}^{+/-}$sheep is expected to eliminate this condition.

Finally, of particular relevance to males with CF - nearly all of whom are infertile due to loss of the vas deferens and often obstruction of the epididymis - $C F T R^{-/-}$male lambs all showed aplasia or severe atrophy of the vas deferens. It is of interest that this loss of genital ducts is evident at birth in the sheep, suggesting an in utero event, which may be obstruction of the ducts with mucus secretions. Further investigation of this model may be useful for understanding the molecular cause of CF male infertility.

Another potential benefit of modeling CF in sheep that has received relatively little attention, but may well become crucial in the future, relates to the early development of the disease. Since CF pathology commences in utero, intrauterine intervention may offer possibilities for effective disease treatment. A sheep model is likely to be more suitable for the development of intrauterine intervention. Because of its similarity in size and anatomy to human, and the length of the gestation, which allows for a long-term in utero observations, the ovine fetus has been extensively used for developing cuttingedge perinatal treatments (reviewed in ref. 33) (34, 35). Availability of an ovine model of CF will open new opportunities to investigate the early disease process, which is impossible to study in humans, and could lead to the development of novel therapeutic strategies.

\section{Methods}

Animals. Domestic sheep (Ovis aries) were used in this study. Female sheep used as embryo recipients were 2-5 years old. They were obtained from the Utah State University Animal Science Farm. CFTR ${ }^{-/}$and $\mathrm{CFTR}^{+/-}$sheep were generated by SCNT using genetically modified SFFs.

Fetal fibroblasts. Primary SFFs were isolated from day 45 Romney fetuses as previously described (36) and cultured in DMEM (high-glucose; Hyclone) supplemented with 15\% FBS (Hyclone) and $100 \mathrm{U} / \mathrm{ml}$ penicillin/streptomycin (Life Technologies) at $38.5^{\circ} \mathrm{C}$ in an atmosphere of $5 \% \mathrm{CO}_{2}$ in air (37). Fetus sex was determine by PCR amplification of the Y chromosome-specific SRY gene (38).

CRISPR/Cas9 targeting vector construction and mutation efficiency detection. The CRISPR/Cas9 gene-targeting vectors were constructed by using the pX330-U6-Chimeric_BB-CBh-hSpCas9 plasmid (Addgene plasmid 42230) as described by Cong et al. (12). The sgRNA/Cas9 targeting sites for each of exon loci of interest were identified by searching for the $\mathrm{G}(\mathrm{N})_{20} \mathrm{GG}$ motifs. The corresponding DNA oligos for each of the targeting sites were synthesized by Integrated DNA Technologies. The final constructs were confirmed by Sanger sequencing. Five micrograms of circular sgRNA/Cas9 vectors were transfected into SFFs using 


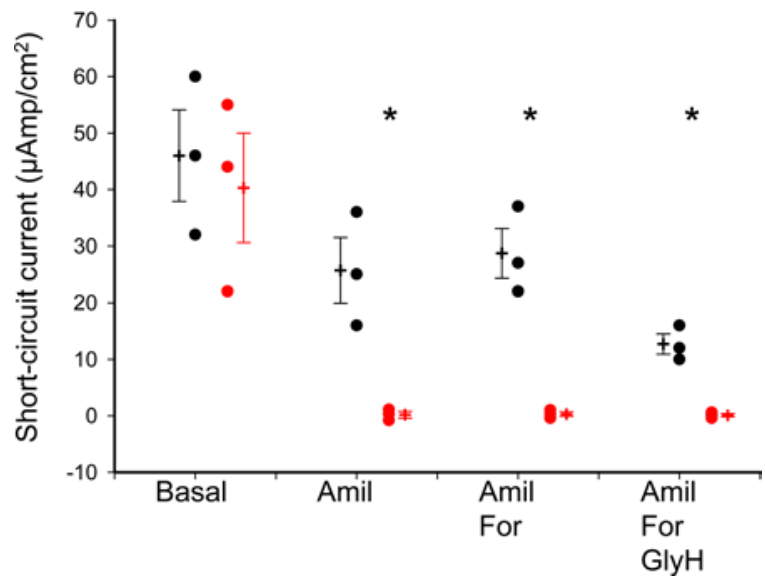

Figure 7. Summary of transepithelial electrophysiology data for WT and CFTR ${ }^{-/-}$sheep tracheal epithelial cell cultures. Air-liquid interface cultures were mounted in Ussing chambers and bathed on both sides with KrebsRinger bicarbonate solution bubbled with $95 \% \mathrm{O}_{2} / 5 \% \mathrm{CO}_{2}$ and maintained at $37^{\circ} \mathrm{C}$. Cultures were maintained under short-circuit conditions and treated sequentially with amiloride (Amil, $100 \mu \mathrm{M}$, apical), forskolin (For, $10 \mu \mathrm{M}$, basolateral), and $\mathrm{GlyH}-101$ (GlyH, $20 \mu \mathrm{M}$, apical). Symbols represent values for individual animals (mean of 6-8 replicates from each animal). Mean \pm SEM for each group is indicated by the crosshair and error bars. ${ }^{*} P<0.05$, between WT and $\mathrm{CFTR}^{-/-}$cultures; unpaired $t$ test.

Amaxa 4D-Nucleofector (program no. EH-100; Lonza). Three days after transfection, cells were harvested for genomic DNA isolation by using a DNeasy Blood \& Tissue Kit (QIAGEN) following the manufacturer's protocol. Each of the targeted genomic loci was PCR amplified from the genomic DNA isolated from SFFs by Phusion High-Fidelity DNA Polymerase (Thermo Fisher Scientific). After digestion with the chosen enzyme (Eco47III for targeting vector 1 or Eco57I for targeting vector 2; Thermo Fisher Scientific), the PCR products were resolved on a 1\% agarose gel and stained with SYBR green dye (Life Technologies). Based on whether the PCR products were fully or partially resistant to digestion by a chosen restriction enzyme (PCR-RFLP assays; indels introduced by sgRNA/Cas9 vectors would abolish the restriction recognition site), indels were detected. To determine gene targeting efficiency by each of the sgRNA/Cas 9 vectors, the relative intensities of the uncut band and cut bands were analyzed by using NIH ImageJ software (1.47p).

Isolation of CFTR-mutated cell colonies. Three days after targeting vector transfection, cells were subjected to single cell cloning by serial dilution in 96 -well plates. Each single cell colony was allowed to grow to near confluence. PCR/RFLP assays and Sanger sequencing were used for the identification of CFTR-mutated colonies.

$S C N T$. Sheep SCNT was performed as described by Yang et al. for goats (39), with minor modifications wherein an aspiration technique was used for oocyte recovery instead of a slicing technique and SOF embryo culture medium was used instead of G1 medium. $C F T R^{-/-}$and $C F T R^{+/-}$fetal fibroblasts were grown to $80 \%-90 \%$ confluence and used as nuclear donor cells for SCNT after 24 hours of serum starvation $(0.5 \%$ FBS). The cloned embryos were cultured in SOF medium for 10-12 hours and then transferred into estrussynchronized recipients as described elsewhere (37).

Identification of $\mathrm{CFTR}^{-/-}$and $\mathrm{CFTR}^{+/-}$fetuses/lambs. PCR/RFLP assay and Sanger sequencing were used for identification of lambs.

OT analysis. We conducted a BLAST search on the sheep nucleotide sequence database with CFTR targeting sequences as the queries to find the genomic sequences with the highest homology using Benchling online software. We chose a total of 19 potential OT sites with the highest sequence homology (scores) to the seed sequences. Specific PCR primers were designed to amplify DNA fragments ranging from $300 \mathrm{bp}$ to $1,000 \mathrm{bp}$ spanning each OT locus. PCR amplification and Sanger sequencing were used for the OT analysis of the genomic DNA isolated from $3 \mathrm{CFTR}^{-/-}$and $3 \mathrm{CFTR}^{+/-}$lambs. PCR primers used for each of the potential OTs are listed in Supplemental Table 2.

Necropsy and histopathologic analysis. Necropsy was performed on all lambs that died or were euthanized. They were examined for gross lesions, and the findings were documented. The following tissue samples were collected and fixed in 10\% neutral buffered formalin for histology: heart, thyroid gland, trachea, lung, thymus, spleen, umbilicus, skeletal muscle, forestomach, intestinal tract, pancreas, liver, kidney, urinary bladder, testis, vas deferens, ovary, uterus, and brain. Formalin-fixed tissue 
sections were processed and embedded in paraffin according to routine histologic techniques. Sections, 5 - $\mu$ m-thick, were stained with H\&E, oil red O stain, PAS stain, or PAS stain after diastase treatment according to standard methods, and examined by light microscopy.

Tracheal cell isolation and primary culture and electrophysiological analysis. Tracheal segments were placed in chilled, sterile culture media with antibiotics immediately after collection and shipped to C. Cotton's laboratory. The culture protocol was based on the method that is routinely used for culture of human airway epithelial cells (17). Tracheal epithelial cells were isolated by injecting the subepithelial space with a collagenase solution (type I; Worthington; $1 \mathrm{mg} / \mathrm{ml}$ in sterile culture media), followed by incubation at $37^{\circ} \mathrm{C}$. After 60 minutes, the segments were removed, and the luminal surface was scraped with a plastic coverslip to recover epithelial sheets. The samples were pooled, centrifuged, and resuspended in Accutase to partially dissociate epithelial sheets into single cells and small clumps. The cells were recovered by centrifugation, resuspended in culture media with antibiotics, and plated on irradiated 3T3 cells $\left(1.7 \times 10^{6} / \mathrm{T}-25\right.$ flask $)$. Cells were then cultured with growth media (F-12/DMEM [3:1]) supplemented with hydrocortisone, insulin, EGF, cholera toxin, adenine, 5\% heat-inactivated FBS, and LY-27632 (Rho-associated kinase inhibitor) as described by Liu et. al. (40). The medium was changed daily. The cells were passaged once and either cryopreserved or seeded onto permeable supports (500,000 cells/filter; Costar 3801) for establishment of air-liquid interface (ALI) primary cultures. Tracheal epithelial cells were seeded onto permeable filters at passage 2 and differentiated into polarized epithelia at ALI. The media used for ALI culture consisted of 1:1 DMEM/F12, supplemented with penicillin/ streptomycin, gentamicin, fungizone, fluconazole, and 2\% Ultroser G. After 35-49 days of ALI culture (i.e., time sufficient to allow differentiation), filters were used for measurements of transepithelial electrophysiology (18). Filters were mounted in a temperature-controlled Ussing chamber that allows for the separate perfusion of the apical and basolateral compartments with a Krebs-Ringer bicarbonate solution, as previously described $(17,18)$. Transepithelial voltage was measured and clamped to 0 to measure short-circuit current. The epithelium was periodically voltage clamped to non-0 values to measure transepithelial resistance.

Statistics. Pregnancy rate, full-term development rate, and birth weight data were analyzed by $\chi^{2}$ test with a 2-tailed $P$ value using GraphPad Prism 6 (GraphPad Software). A difference was considered significant when the $P$ value was less than 0.05 . Short-circuit current and resistance values for non-CF and $\mathrm{CF}$ sheep tracheal epithelial cell cultures were analyzed by unpaired $t$ test. A difference was considered significant when the $P$ value was less than 0.05 .

Study approval. All animal studies were approved and monitored by the IACUC at Utah State University (IACUC protocol 2410) and conformed to NIH guidelines.

\section{Author contributions}

$\mathrm{AH}, \mathrm{KLW}$, and IAP conceived and managed the study. ZF, IVP, and ZW designed and/or carried out the CRISPR/Cas9 targeting. ZF, MR, and QM conducted SCNT experiments. AJVW carried out the necropsies and histopathological analysis of the CF lambs. CC and CD performed electrophysiological analysis of trachea epithelial cells. AH, ZF, IVP, AJVW, CC, and IAP wrote the manuscript.

\section{Acknowledgments}

We thank Rusty Stott for performing embryo transfers procedures, and David Forrester and Angela Robinson for excellent assistance with animal care. This work was supported by the Utah Agricultural Experiment Station (UTAO1328) and the Cystic Fibrosis Foundation (award POLEJA17XX0).

Address correspondence to: Irina A. Polejaeva, Department of Animal, Dairy and Veterinary Sciences, Utah State University, Logan, Utah 84322-4815, USA. Email: irina.polejaeva@usu.edu. Or to: Ann Harris, Department of Genetics and Genome Sciences, Case Western Reserve University School of Medicine, Cleveland, Ohio 44106-4955, USA. Email: ann.harris@case.edu.

1. Ratjen F, Grasemann H. New therapies in cystic fibrosis. Curr Pharm Des. 2012;18(5):614-627.

2. Lavelle GM, White MM, Browne N, McElvaney NG, Reeves EP. Animal models of cystic fibrosis pathology: phenotypic parallels and divergences. Biomed Res Int. 2016;2016:5258727.

3. Harris A. Towards an ovine model of cystic fibrosis. Hum Mol Genet. 1997;6(13):2191-2194.

4. Thorburn GD, Harding R. Textbook of Fetal Physiology. Oxford, United Kingdom: Oxford University Press; 1994.

5. Cotton CU, Lawson EE, Boucher RC, Gatzy JT. Bioelectric properties and ion transport of airways excised from adult and fetal 
sheep. J Appl Physiol Respir Environ Exerc Physiol. 1983;55(5):1542-1549.

6. Broackes-Carter FC, Mouchel N, Gill D, Hyde S, Bassett J, Harris A. Temporal regulation of CFTR expression during ovine lung development: implications for CF gene therapy. Hum Mol Genet. 2002;11(2):125-131.

7. Mouchel N, Broackes-Carter F, Harris A. Alternative 5' exons of the CFTR gene show developmental regulation. Hum Mol Genet. 2003;12(7):759-769.

8. Trezise AE, Chambers JA, Wardle CJ, Gould S, Harris A. Expression of the cystic fibrosis gene in human foetal tissues. Hum Mol Genet. 1993;2(3):213-218.

9. Harris A, Chalkley G, Goodman S, Coleman L. Expression of the cystic fibrosis gene in human development. Development. 1991;113(1):305-310.

10. Williams SH, Sahota V, Palmai-Pallag T, Tebbutt SJ, Walker J, Harris A. Evaluation of gene targeting by homologous recombination in ovine somatic cells. Mol Reprod Dev. 2003;66(2):115-125.

11. Schnieke AE, et al. Human factor IX transgenic sheep produced by transfer of nuclei from transfected fetal fibroblasts. Science. 1997;278(5346):2130-2133.

12. Cong L, et al. Multiplex genome engineering using CRISPR/Cas systems. Science. 2013;339(6121):819-823.

13. Dawson AJ, King TJ, Wilmut I, Harkness LM, Kelly BG, Rhind SM. Immunohistochemical characterization of cloned lamb nephropathy. J Histochem Cytochem. 2004;52(12):1657-1664.

14. Rhind SM, et al. Cloned lambs — lessons from pathology. Nat Biotechnol. 2003;21(7):744-745.

15. Chavatte-Palmer $\mathrm{P}$, et al. Clinical, hormonal, and hematologic characteristics of bovine calves derived from nuclei from somatic cells. Biol Reprod. 2002;66(6):1596-1603.

16. Kato Y, Tani T, Tsunoda Y. Cloning of calves from various somatic cell types of male and female adult, newborn and fetal cows. J Reprod Fertil. 2000;120(2):231-237.

17. Cotton C, et al. Large-scale expansion of primary cultures of nasal epithelial cells from CF patients [North American Cystic Fibrosis Conference abstract 174A]. Pediatr Pulm. 2014:49(suppl 38):277.

18. Veizis EI, Carlin CR, Cotton CU. Decreased amiloride-sensitive Na+ absorption in collecting duct principal cells isolated from BPK ARPKD mice. Am J Physiol Renal Physiol. 2004;286(2):F244-F254.

19. Campbell KH, McWhir J, Ritchie WA, Wilmut I. Sheep cloned by nuclear transfer from a cultured cell line. Nature. 1996;380(6569):64-66.

20. Wilmut I, Schnieke AE, McWhir J, Kind AJ, Campbell KH. Viable offspring derived from fetal and adult mammalian cells. Nature. 1997;385(6619):810-813.

21. Harris A. Towards an ovine model of cystic fibrosis. Hum Mol Genet. 1997;6(13):2191-2194.

22. Jinek M, Chylinski K, Fonfara I, Hauer M, Doudna JA, Charpentier E. A programmable dual-RNA-guided DNA endonuclease in adaptive bacterial immunity. Science. 2012;337(6096):816-821.

23. Rogers CS, et al. Disruption of the CFTR gene produces a model of cystic fibrosis in newborn pigs. Science. 2008;321(5897):1837-1841.

24. Sun X, et al. Disease phenotype of a ferret CFTR-knockout model of cystic fibrosis. J Clin Invest. 2010;120(9):3149-3160.

25. Stoltz DA, et al. Intestinal CFTR expression alleviates meconium ileus in cystic fibrosis pigs. J Clin Invest. 2013;123(6):2685-2693.

26. Gordon JI, Elshourbagy N, Lowe JB, Liao WS, Alpers DH, Taylor JM. Tissue specific expression and developmental regulation of two genes coding for rat fatty acid binding proteins. J Biol Chem. 1985;260(4):1995-1998.

27. Rogers CS, et al. Production of CFTR-null and CFTR-DeltaF508 heterozygous pigs by adeno-associated virus-mediated gene targeting and somatic cell nuclear transfer. J Clin Invest. 2008;118(4):1571-1577.

28. Stoltz DA, et al. Cystic fibrosis pigs develop lung disease and exhibit defective bacterial eradication at birth. Sci Transl Med. 2010;2(29):29ra31.

29. Reid CJ, Hyde K, Ho SB, Harris A. Cystic fibrosis of the pancreas: involvement of MUC6 mucin in obstruction of pancreatic ducts. Mol Med. 1997;3(6):403-411

30. Abu-El-Haija M, et al. Pancreatic damage in fetal and newborn cystic fibrosis pigs involves the activation of inflammatory and remodeling pathways. Am J Pathol. 2012;181(2):499-507.

31. Epstein NE. Commentary on: Laminectomy plus fusion versus laminectomy alone for lumbar spondylolisthesis by Ghogawala Z, Dziura J, Butler WE, Dai F, Terrin N, Magge SN, et al. NEJM 2016;374 (15):1424-34. Surg Neurol Int. 2016;7(Suppl 25):S644-S647.

32. Lamireau T, Monnereau S, Martin S, Marcotte JE, Winnock M, Alvarez F. Epidemiology of liver disease in cystic fibrosis: a longitudinal study. J Hepatol. 2004;41(6):920-925.

33. Morel O, Laporte-Broux B, Tarrade A, Chavatte-Palmer P. The use of ruminant models in biomedical perinatal research. Theriogenology. 2012;78(8):1763-1773.

34. Meuli M, et al. In utero repair of experimental myelomeningocele saves neurological function at birth. J Pediatr Surg. 1996;31(3):397-402.

35. Eggink AJ, et al. In utero repair of an experimental neural tube defect in a chronic sheep model using biomatrices. Fetal Diagn Ther. 2005;20(5):335-340.

36. Keefer CL, et al. Generation of dwarf goat (Capra hircus) clones following nuclear transfer with transfected and nontransfected fetal fibroblasts and in vitro-matured oocytes. Biol Reprod. 2001;64(3):849-856.

37. Yang M, Perisse I, Fan Z, Regouski M, Meyer-Ficca M, Polejaeva IA. Increased pregnancy losses following serial somatic cell nuclear transfer in goats [published online ahead of print May 17, 2018]. Reprod Fertil Dev. https://doi.org/10.1071/RD17323

38. Saberivand A, Ahsan S. Sex determination of ovine embryos by SRY and amelogenin (AMEL) genes using maternal circulating cell free DNA. Anim Reprod Sci. 2016;164:9-13.

39. Yang $M$, et al. Oocytes from small and large follicles exhibit similar development competence following goat cloning despite their differences in meiotic and cytoplasmic maturation. Theriogenology. 2016;86(9):2302-2311.

40. Liu X, Luo M, Zhang L, Ding W, Yan Z, Engelhardt JF. Bioelectric properties of chloride channels in human, pig, ferret, and mouse airway epithelia. Am J Respir Cell Mol Biol. 2007;36(3):313-323. 